\title{
Video Article \\ Validating Whole Genome Nanopore Sequencing, using Usutu Virus as an Example
}

\author{
Bas B. Oude Munnink ${ }^{1}$, David F. Nieuwenhuijse ${ }^{1}$, Reina S. Sikkema ${ }^{1}$, Marion Koopmans ${ }^{1}$ \\ ${ }^{1}$ ErasmusMC, Department of Viroscience, WHO Collaborating Centre for Arbovirus and Viral Hemorrhagic Fever Reference and Research, Erasmus University Medical \\ Center
}

Correspondence to: Bas B. Oude Munnink at b.oudemunnink@erasmusmc.nl

URL: https://www.jove.com/video/60906

DOI: doi:10.3791/60906

Keywords: Genetics, Issue 157, nanopore, sequencing, R10 flowcell, USUV, arboviruses, whole genome sequencing

Date Published: 3/11/2020

Citation: Oude Munnink, B.B., Nieuwenhuijse, D.F., Sikkema, R.S., Koopmans, M. Validating Whole Genome Nanopore Sequencing, using Usutu Virus as an Example. J. Vis. Exp. (157), e60906, doi:10.3791/60906 (2020).

\section{Abstract}

Whole genome sequencing can be used to characterize and to trace viral outbreaks. Nanopore-based whole genome sequencing protocols have been described for several different viruses. These approaches utilize an overlapping amplicon-based approach which can be used to target a specific virus or group of genetically related viruses. In addition to confirmation of the virus presence, sequencing can be used for genomic epidemiology studies, to track viruses and unravel origins, reservoirs and modes of transmission. For such applications, it is crucial to understand possible effects of the error rate associated with the platform used. Routine application in clinical and public health settings require that this is documented with every important change in the protocol. Previously, a protocol for whole genome Usutu virus sequencing on the nanopore sequencing platform was validated (R9.4 flowcell) by direct comparison to Illumina sequencing. Here, we describe the method used to determine the required read coverage, using the comparison between the R10 flow cell and Illumina sequencing as an example.

\section{Video Link}

The video component of this article can be found at https://www.jove.com/video/60906/

\section{Introduction}

Fast developments in third generation sequence technologies allows us to move forward towards close to real-time sequencing during viral outbreaks. This timely availability of genetic information can be useful to determine the origin and evolution of viral pathogens. Gold standards in the fields of next generation sequencing however, are still the second-generation sequencers. These techniques rely on specific and timeconsuming techniques like clonal amplification during an emulsion PCR or clonal bridge amplification. The third-generation sequencers are cheaper, hand-held and come with simplified library preparation methodologies. Especially the small size of the sequence device and the low purchase price makes it an interesting candidate for deployable, fieldable sequencing. This could for instance be seen during the Ebola virus outbreak in Sierra Leone and during the ongoing arbovirus outbreak investigations in Brazil ${ }^{1,2,3}$. However, the reported high error rate ${ }^{4}$ might limit the applications for which nanopore sequencing can be used.

Nanopore sequencing is evolving quickly. New products are available in the market on a regular basis. Examples of this are for instance the 1D squared kits which enables sequencing of both strands of the DNA molecule, thereby boosting the accuracy of the called bases ${ }^{5}$ and the development of the R10 flow cell which measures the change in current at two different instances in the pore ${ }^{6}$. In addition, improved bioinformatic tools like improvements in basecalling will improve the accuracy of basecalling ${ }^{7}$. One of the most frequently used basecallers, (e.g., Albacore), has been updated at least 12 times in a 9-month time period ${ }^{5}$. Recently, the manufacturer also released a novel basecaller called flipflop, which is implemented in the default nanopore software ${ }^{8}$. Together, all of these improvements will lead to more accurate sequences and will decrease the error rate of the nanopore sequencer.

Usutu virus (USUV) is a mosquito-borne arbovirus of the family Flaviviridae and it has a positive-stranded RNA genome of around 11,000 nucleotides. USUV mainly affects great grey owls and blackbirds ${ }^{9,10}$, although other bird species are also susceptible to USUV infection ${ }^{11}$. Recently, USUV was also identified in rodents and shrews although their potential role in transmission of the virus remains unknown ${ }^{12}$. In humans, asymptomatic infections have been described in blood donors ${ }^{13,14,15,16}$ while USUV infections also have been reported to be associated with encephalitis or meningo-encephalitis ${ }^{17,18}$. In the Netherlands, USUV was first detected in wild birds in $2016^{10}$ and in asymptomatic blood donors in $2018^{14}$. Since the initial detection of USUV, outbreaks have been reported during the subsequent years and surveillance, including whole genome sequencing, is currently ongoing to monitor the emerge and spread of an arbovirus in a previously naïve population.

Similar to what has been described for other viruses, such as Ebola virus, Zika virus and yellow fever virus ${ }^{3,19,20}$, we have developed a primer set to sequence full length USUV ${ }^{21}$. This polymerase chain reaction (PCR)-based approach allows for the recovery of full length USUV genomes from highly host-contaminated sample types like brain samples in samples up to a Ct value of around 32. Benefits of an amplicon-based sequencing approach are a higher sensitivity compared to metagenomic sequencing and a higher specificity. Limitations of using an amplicon- 
based approach are that the sequences should be similar in order to design primers fitting all strains and that primers are designed on our current knowledge about the virus diversity.

Given the constant developments and improvements in third generation sequencing, there is a need to evaluate the error rate of the sequencer on a regular basis. Here, we describe a method to evaluate the performance of nanopore directly against Illumina sequencing using USUV as an example. This method is applied to sequences generated with the latest R10 flow cell and basecalling is performed with the latest version of the flip-flop basecaller.

\section{Protocol}

NOTE: List of software tools to be used: usearch v11.0.667; muscle v3.8.1551; porechop 0.2.4; cutadapt 2.5; minimap2 2.16-r922; samtools 1.9; trimmomatic 0.39; bbmap 38.33; spades v3.13.1; kma-1.2.8

\section{Primer design}

1. Start with downloading or retrieving a set of relevant reference whole genome sequences from public or private data collections. For instance, retrieve all full length USUV genomes (taxid64286) from the NCBI database ${ }^{22}$. USUV encodes a genome of around 11,000 nucleotides so only retrieve the sequences with a sequence length of 8,000-12,000 nucleotides. Do this using the following search entry: - taxid64286[Organism:noexp] AND 8000[SLEN]:12000[SLEN].

1. Click on Send to | Complete Record | File; use Format = FASTA and create the File.

2. To downsize the set of reference sequences, remove duplicate sequences or sequences with over $99 \%$ nucleotide identity from the dataset. Do this using the cluster fast option from usearch ${ }^{23}$. On the command line enter: - usearch -cluster_fast All_USUV.fasta -id 0.99 -centroids All_USUV_dedup.fasta

3. To generate the primers, sequences need to be aligned. This is done using MUSCLE ${ }^{24}$. On the command line enter: - muscle -in All_USUV_dedup.fasta -out AII_USUV_dedup_aligned.fasta -log log_muscle.txt NOTE: It is essential to manually inspect the alignment to check for discrepancies. These can be manually corrected if needed and the ends can be trimmed according to the length of most whole genome sequences.

4. Primal is used to make a draft selection of the primers which can be used for full length amplicon sequencing ${ }^{19}$. Upload the alignment to the primal website (http://primal.zibraproject.org/) and select the preferred amplicon length and overlap length between the different amplicons. Go to primal.zibraproject.org, fill in the Scheme name, upload the aligned fasta file, select the amplicon length, overlap size, and generate the scheme.

5. Align the complete set of available complete USUV sequences (not the downsized or deduplicated set). On the command line enter: - muscle -in All_USUV.fasta -out All_USUV_aligned.fasta -log log_muscle.txt NOTE: Map the generated primers against the complete alignment (do not use the deduplicated alignment), manually correct errors and include a maximum of 5 degenerative primer positions.

\section{Multiplex PCR}

1. Perform the multiplex PCR using the designed primers and nanopore and Illumina sequencing. The multiplex PCR for USUV was performed as previous described ${ }^{19,21}$.

2. Perform basecalling with flip-flop version 3.0.6.6+9999d81.

\section{Data analysis to generate consensus sequences from nanopore data}

1. Several samples can be multiplexed on a single nanopore sequencing run. After performing the sequence run, demultiplex the nanopore data. Use Porechop ${ }^{25}$ for this. To prevent contamination and enhance accuracy, use the require_two_barcodes flag. On the command line enter:

- porechop -i Run_USUV.fastq -o Run_USUV_demultiplex --require_two_barcodes

2. After demultiplexing, remove primer sequences (indicated in the file Primers_Usutu.fasta in both orientations) using cutadapt ${ }^{26}$. In addition, remove sequences with a length shorter than 75 nucleotides. The primers have to be removed since they can introduce artificial biases in the consensus sequence. On the command line enter: - cutadapt -b file:Primers_USUV.fasta -o BC01_trimmed.fastq BC01.fastq -m 75

3. Demultiplexed sequence reads can be mappe $\overline{-}$ against a panel of distinct reference strains using minimap $2^{27}$ and a consensus sequence can be generated using samtools ${ }^{28}$. Follow the example below which shows the procedure of a reference-based alignment and the consensus sequence generation of one sample: BC01. On the command line enter:

- minimap2 -ax map-ont Random_Refs_USUV.fasta BC01_trimmed.fastq > BC01.bam

- samtools sort BC01.bam > BC01_sorted.bam

- bcftools mpileup -Ou -f Random_Refs_USUV.fasta BC01_sorted.bam | bcftools call -mv -Oz -o BC01.vcf.gz

- bcftools index BC01.vcf.gz

- cat Random_Refs_USUV.fasta| bcftools consensus BC01.vcf.gz > BC01_consensus.fasta

4. For reference-base $\bar{d}$ alignments it is essential that a closely related reference sequence is used. Therefore, perform a BlastN search with the generated consensus sequence to identify the closest reference strain. After that, repeat the reference-based alignment with the closest reference strain as reference (step 3.3 and 3.4). On the command line enter:

- minimap2 -ax map-ont Ref_USUV_BC01.fasta BC01_trimmed.fastq > BC01_ref.bam

- samtools sort BC01 ref.bam > BC01 sorted ref.bam

- bcftools mpileup -Ou -f Ref_USUV_BC01.fasta BC01_sorted_ref.bam | bcftools call -mv-Oz -o BC01_ref.vcf.gz 
- bcftools index BC01 ref.vcf.gz

- cat Ref_USUV_BC01.fasta | bcftools consensus BC01_ref.vcf.gz > BC01_ref_consensus.fasta

\section{Analysis of the Illumina data}

1. These sequences are automatically demultiplexed after sequencing. Reads can be quality controlled using trimmomatic ${ }^{29}$. For paired-end Illumina sequences, use the commonly used cut-off median PHRED score of 33 and a minimal read length of 75 to get accurate, high quality reads. On the command line enter:

- trimmomatic PE -phred33 9_S9_L001_R1_001.fastq.gz 9_S9_L001_R2_001.fastq.gz 9_1P.fastq9_1U.fastq 9_2P.fastq 9_2U.fastq LEADING:3 TRAILING:3 SLIDINGWINDOW:3:15 MINLEN:75

2. Remove primers (indicated in the file Primers_Usutu.fasta in both orientations), since they can introduce artificial biases, using cutadapt ${ }^{26}$. In addition, remove sequences with a length shorter than 75 nucleotides using the commands below. On the command line enter: - cutadapt -b o 9_1P_trimmed.fastq -p 9_2P_trimmed.fastq 9_1P.fastq 9_2P.fastq $-m 75$

3. Before de novo assembly, the sequence reads can be normalized for an even coverage across the genome. This is essential since de novo assemblers like SPAdes take the read coverage into account when assembling sequence reads. Normalize reads to a read coverage of 50 using BBNorm from the BBMap package ${ }^{30}$. On the command line enter:

- bbmap/bbnorm. sh target $=50$ in=9_1P_trimmed.fastq in2=9_2P_trimmed.fastq out=Sample9_FW_norm.fastq out2=Sample9_RE_norm.fastq

4. The normalized reads are de novo assembled using SPAdes ${ }^{31}$. Default settings are used for the assembly using all different kmers $(21,33$, 55, 77, 99 and 127). On the command line enter: - spades.py -k 21,33,55,77,99,127 -o Sample9 -1 Sample9.qc.f.fq -2 Sample9.qc.r.fq

5. Map the QC reads against the obtained consensus sequence using minimap2 and programs like Geneious, Bioedit or Ugene to curate the alignment. It is important to check the beginning and the end of the contig.

1. Align the $Q C$ reads against the obtained consensus sequencing using minimap2.

2. Import the alignment in Geneious/Bioedit/UGene.

3. Manually inspect, correct and curate especially the beginning and the end of the genome.

\section{Determining the required read coverage to compensate for the error profile in nanopore sequencing using Illumina data as gold standard}

1. Select sequence reads mapping to one amplicon, in this case amplicon 26 . Subsequently, map the nanopore reads against this amplicon using minimap2. Use Samtools to select only the reads mapping to amplicon 26 and to convert the bam file into fastq. On the command line enter:

- minimap2 -ax map-ont -m 150 Amplicon26.fasta BC01_trimmed.fastq > BC01.bam

- samtools view -b -F 4 BC01.bam > BC01_mapped.bam

- samtools bam2fq BC01_mapped.bam | seqtk seq - -> BC01_mapped.fastq

2. Randomly select subsets of for instance 200 sequence reads one thousand times. For example, changing it to 10 will result in the random selection of one thousand times a subset of 10 sequence reads. The script is provided as Supplementary File 1. On the command line enter:

- python Random_selection.py

3. All randomly selected sequence reads are aligned to amplicon 26 . Use $\mathrm{KMA}^{32}$ to map the sequence reads and to immediately generate a consensus sequence. Use optimized settings for nanopore sequencing, indicated by the -bcNano flag. On the command line enter:

- kma index -i Amplicon26.fasta

- for file in random sample*; do

- sampleID $=\$\{$ file $\%$.fastq\}

- kma -i \$\{sampleID\}.fastq -o \$\{sampleID\} -t_db Amplicon26.fasta -mem_mode -mp 5 -mrs 0.0 -bcNano

- done

4. Inspect the generated consensus sequences on the command line using:

- cat ${ }^{*}$.fsa > All_genomes.fsa

- minimap2 -ax map-ont Amplicon26.fasta All_genomes.fsa > All_genomes.bam

- samtools sort All_genomes.bam > All_genomes_sorted.bam

- samtools stats All_genomes_sorted.bam > stats.txt

1. The error rate is displayed in the stats.txt under the heading error rate \#mismatches / bases mapped. Display it on the screen with the following command: - grep ${ }^{\wedge} S N$ stats.txt | cut -f 2-

2. The amount of indels is displayed under the heading \#Indels per cycle. Display it on the screen with the following command: - grep ${ }^{\wedge} / C$ stats.txt | cut - $f$ 2- 


\section{Representative Results}

Recently, a new version of the flow cell version (R10) was released and offered improvements to the basecaller used to convert the electronic current signal to DNA sequences (so-called flip-flop basecaller). Therefore, we have re-sequenced USUV from brain tissue of an USUVpositive owl which was previously sequenced on a R9.4 flow cell and on an Illumina Miseq instrument ${ }^{21}$. Here, we described the method used to determine the required read coverage for reliable consensus calling by direct comparison to Illumina sequencing.

Using the newer flow cell in combination with the basecaller flip-flop we show that a read coverage of $40 x$ results in identical results as compared to Illumina sequencing. A read coverage of $30 x$ results in an error rate of $0.0002 \%$ which corresponds to one error in every 585,000 nucleotides sequenced, while a read coverage of $20 x$ results in one error in every 63,529 nucleotides sequenced. A read coverage of $10 x$ results in one error in every 3,312 nucleotides sequenced, meaning that over three nucleotides per full USUV genome are being called wrong. With a read coverage above $30 x$, no indels were observed. A read coverage of $20 x$ resulted in the detection of one indel position while a read coverage of $10 x$ resulted in indels in 29 positions. An overview of the error rate using different read coverage cut-offs is shown in Table 1.

\begin{tabular}{|c|c|c|c|c|c|c|c|c|c|}
\hline Coverage & \begin{tabular}{|l|} 
Errors \\
iteration 1
\end{tabular} & \begin{tabular}{|l|} 
Error rate \\
iteration 1
\end{tabular} & Indels: & \begin{tabular}{|l|} 
Errors \\
iteration 2
\end{tabular} & \begin{tabular}{|l|} 
Error rate \\
iteration 2
\end{tabular} & Indels: & \begin{tabular}{|l|} 
Errors \\
iteration 3
\end{tabular} & \begin{tabular}{|l|} 
Error rate \\
iteration 3
\end{tabular} & Indels: \\
\hline $10 x$ & 100 & $0.0274 \%$ & 4 & 116 & $0.0297 \%$ & 18 & 110 & $0.0282 \%$ & 7 \\
\hline $20 x$ & 4 & $0.0010 \%$ & 0 & 6 & $0.0015 \%$ & 1 & 7 & $0.0018 \%$ & 0 \\
\hline $30 x$ & 2 & $0.0005 \%$ & 0 & 0 & $0.0000 \%$ & 0 & 0 & $0.0000 \%$ & 0 \\
\hline $40 x$ & 0 & $0.0000 \%$ & 0 & 0 & $0.0000 \%$ & 0 & 0 & $0.0000 \%$ & 0 \\
\hline $50 x$ & 0 & $0.0000 \%$ & 0 & 0 & $0.0000 \%$ & 0 & 0 & $0.0000 \%$ & 0 \\
\hline
\end{tabular}

Table 1: Overview of the error rate of nanopore sequencing. Each iteration represents one thousand random samples.

Supplementary File 1: Random selection. Please click here to view this file (Right click to download).

\section{Discussion}

Nanopore sequencing is constantly evolving and therefore there is a need for methods to monitor the error rate. Here, we describe a workflow to monitor the error rate of the nanopore sequencer. This can be useful after the release of a new flow cell, or if new releases of the basecalling are released. However, this can also be useful for users who want to set-up and validate their own sequencing protocol.

Different software and alignment tools can yield different results ${ }^{33}$. In this manuscript, we aimed to use freely available software packages which are commonly used, and which have clear documentation. In some cases, preference might be given to commercial tools, which generally have a more user-friendly interfaces but have to be paid for. In the future, this method can be applied to the same sample in case big modifications in sequence technology or basecalling software are introduced Preferentially this should be done after each update of the basecaller or flowcell, however given the speed of the current developments this can be also been done only after major updates.

The reduction in the error rate in sequencing allows for a higher number of samples to be multiplexed. Thereby, nanopore sequencing is getting closer to replacing conventional real time PCRs for diagnostic assays, which is already the case for influenza virus diagnostics. In addition, the reduction of the error rate increases the usability of this technique sequencing, for instance for the determination of minor variants and for highthroughput unbiased metagenomic sequencing.

A critical step in the protocol is that close, reliable reference sequences need to be available. The primers are based on the current knowledge about virus diversity and might need to be updated every once in a while. Another critical point when setting up an amplicon-based sequencing approach is the balancing of the primer concentration to get an even balance in amplicon depth. This enables the multiplexing of more samples on a sequence run and results in a significant cost reduction.

\section{Disclosures}

The authors have nothing to disclose.

\section{Acknowledgments}

This work has received funding from the European Union's Horizon 2020 research and innovation programme under grant agreement No. 643476 (COMPARE).

\section{References}

1. Faria, N. R. et al. Establishment and cryptic transmission of Zika virus in Brazil and the Americas. Nature. 546 (7658), $406-410$ (2017).

2. Bonaldo, M. C. et al. Genome analysis of yellow fever virus of the ongoing outbreak in Brazil reveals polymorphisms. Memórias do Instituto Oswaldo Cruz. 112 (6), 447-451 (2017).

3. Faria, N. R. et al. Genomic and epidemiological monitoring of yellow fever virus transmission potential. bioRxiv. 299842 (2018). 
4. Magi, A., Giusti, B., Tattini, L. Characterization of MinION nanopore data for resequencing analyses. Briefings in Bioinformatics. 18 (6), bbw077 (2016).

5. Rang, F. J., Kloosterman, W.P., de Ridder, J. From squiggle to basepair: computational approaches for improving nanopore sequencing read accuracy. Genome Biology. 19 (1), 90 (2018).

6. Nanopore Store, R10 flow cells. https://store.nanoporetech.com/flowcells/spoton-flow-cell-mk-i-r10.html (2019).

7. Wick, R. R., Judd, L. M., Holt, K.E. Performance of neural network basecalling tools for Oxford Nanopore sequencing. Genome Biology. 20 (1), 129 (2019)

8. GitHub - nanoporetech/flappie: Flip-flop basecaller for Oxford Nanopore reads. https://github.com/nanoporetech/flappie [software] (2019).

9. Lühken, R. et al. Distribution of Usutu Virus in Germany and Its Effect on Breeding Bird Populations. Emerging Infectious Diseases. 23 (12), 1994-2001 (2017).

10. Cadar, D. et al. Widespread activity of multiple lineages of Usutu virus, Western Europe, 2016. Eurosurveillance. 22 (4) (2017).

11. Becker, N. et al. Epizootic emergence of Usutu virus in wild and captive birds in Germany. PLoS ONE. 7 (2) (2012).

12. Diagne, M. et al. Usutu Virus Isolated from Rodents in Senegal. Viruses. 11 (2), 181 (2019).

13. Bakonyi, T. et al. Usutu virus infections among blood donors, Austria, July and August 2017 - Raising awareness for diagnostic challenges. Eurosurveillance. 22 (41) (2017).

14. Zaaijer, H. L., Slot, E., Molier, M., Reusken, C.B.E.M., Koppelman, M.H.G.M. Usutu virus infection in Dutch blood donors. Transfusion. trf.15444 (2019).

15. Cadar, D. et al. Blood donor screening for West Nile virus (WNV) revealed acute Usutu virus (USUV) infection, Germany, September 2016. Eurosurveillance. 22 (14), 30501 (2017).

16. Pierro, A. et al. Detection of specific antibodies against West Nile and Usutu viruses in healthy blood donors in northern Italy, 2010-2011. Clinical Microbiology and Infection. 19 (10), E451-E453 (2013).

17. Pecorari, M. et al. First human case of Usutu virus neuroinvasive infection, Italy, August-September 2009. Euro surveillance: bulletin européen sur les maladies transmissibles = European Communicable Disease Bulletin. 14 (50) (2009).

18. Simonin, Y. et al. Human Usutu Virus Infection with Atypical Neurologic Presentation, Montpellier, France, 2016. Emerging Infectious Diseases. 24 (5), 875-878 (2018).

19. Quick, J. et al. Multiplex PCR method for MinION and Illumina sequencing of Zika and other virus genomes directly from clinical samples. Nature Protocols. 12 (6), 1261-1276 (2017).

20. Quick, J. et al. Real-time, portable genome sequencing for Ebola surveillance. Nature. 530 (7589), $228-232$ (2016).

21. Oude Munnink, B. B. et al. Towards high quality real-time whole genome sequencing during outbreaks using Usutu virus as example. Infection, Genetics and Evolution. 73, 49-54 (2019).

22. Benson, D. A., Karsch-Mizrachi, I., Lipman, D.J., Ostell, J., Sayers, E.W. GenBank. Nucleic Acids Research. 38 (Database issue), D46-51 (2010).

23. Edgar, R. C. Search and clustering orders of magnitude faster than BLAST. Bioinformatics. 26 (19), $2460-2461$ (2010).

24. Edgar, R. C. MUSCLE: multiple sequence alignment with high accuracy and high throughput. Nucleic Acids Research. 32 (5), $1792-1797$ (2004).

25. R. R. Wick GitHub - rrwick/Porechop: adapter trimmer for Oxford Nanopore reads. https://github.com/rrwick/porechop [software] (2018).

26. Martin, M. Cutadapt removes adapter sequences from high-throughput sequencing reads. EMBnet.journal. 17 (1), 10 (2011).

27. Li, H. Minimap2: pairwise alignment for nucleotide sequences. Bioinformatics. 34 (18), 3094-3100 (2018).

28. Li, H. et al. The Sequence Alignment/Map format and SAMtools. Bioinformatics. 25 (16), 2078-2079 (2009).

29. Bolger, A. M., Lohse, M., Usadel, B. Trimmomatic: a flexible trimmer for Illumina sequence data. Bioinformatics (Oxford, England). 30 (15), 2114-20 (2014).

30. BBMap download | SourceForge.net. https://sourceforge.net/projects/bbmap/ [software] (2019).

31. Bankevich, A. et al. SPAdes: a new genome assembly algorithm and its applications to single-cell sequencing. Journal of Computational Biology. 19 (5), 455-477 (2012).

32. Clausen, P. T. L. C., Aarestrup, F.M., Lund, O. Rapid and precise alignment of raw reads against redundant databases with KMA. BMC Bioinformatics. 19 (1), 307 (2018).

33. Brinkmann, A. et al. Proficiency Testing of Virus Diagnostics Based on Bioinformatics Analysis of Simulated In Silico High-Throughput Sequencing Data Sets. Journal of Clinical Microbiology. 57 (8) (2019). 